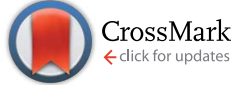

Cite this: RSC Adv., 2014, 4, 36101

Received 21st May 2014

Accepted 11th August 2014

DOI: $10.1039 / \mathrm{c} 4 \mathrm{ra0} 4795 \mathrm{~h}$

www.rsc.org/advances

\title{
Efficient multiphoton upconversion and synthesis route dependent emission tunability in $\mathrm{GdPO}_{4}: \mathrm{Ho}^{3+}, \mathrm{Yb}^{3+}$ nanocrystals $\uparrow$
}

\begin{abstract}
Vineet Kumar, Poonam Rani, Dinesh Singh and Santa Chawla*
Double rare earth ion doped $\mathrm{GdPO}_{4}$ nanocrystals with multiform morphologies, such as nanoparticles, nanospheres, and nanorods have been synthesized via four different routes namely a facile solid-state reaction (SSR) method, low temperature co-precipitation (CPP), pechini type sol-gel (SG) and an auto combustion process (ACP). All the methods produce crystalline nanoparticles with dimensions ranging from a few $\mathrm{nm}$ to tens of $\mathrm{nm}$ but with different morphologies. We systematically investigated the photoluminescence properties of $\mathrm{GdPO}_{4}: \mathrm{Ho}^{3+}, \mathrm{Yb}^{3+}$ nanocrystals under IR (980 nm) excitation and observed that the emission colour is green for the sample synthesized by the SG route whereas all other samples predominantly emit in the red due to efficient energy transfer from $\mathrm{Yb}^{3+}$ to emitter $\mathrm{Ho}^{3+}$ in the $\mathrm{GdPO}_{4}$ host. The upconversion was found to be a two photon process in $\mathrm{GdPO}_{4}: \mathrm{Ho}^{3+}, \mathrm{Yb}^{3+}$ nanocrystals and synthesis route dependent emission tunability suggests different intra level phonon relaxation routes.
\end{abstract}

\section{Introduction}

Photon upconversion (anti-Stokes emission) is a multi photon process in which absorption of infrared (IR) photons leads to visible emission. Efficient up conversion phosphors are scarce and mostly fluoride hosts such as $\mathrm{NaYF}_{4} / \mathrm{NaGdF}_{4}$ doped with emitters $\mathrm{Er}^{3+}, \mathrm{Tm}^{3+}, \mathrm{Ho}^{3+}$ and $\mathrm{Yb}^{3+}$ as sensitizers ${ }^{1-4}$ are chosen due to their low phonon energy. Fluoride materials are reactive, difficult to produce through facile routes and mostly synthesized in organic solvents with emission of fluorine components that are hazardous. It is very important to explore non reactive upconversion (UC) inorganic phosphor hosts for use in applications such as direct deposition on a device e.g., depositing a phosphor layer on a Si solar cell for solar spectrum conversion or on a IR LED chip for making a blue $\left(\mathrm{Tm}^{3+}\right.$ doped), green $\left(\mathrm{Er}^{3+}\right.$ doped), red ( $\mathrm{Er}^{3+} / \mathrm{Ho}^{3+}$ doped) LED. Synthesis of such phosphor has to be cost effective through easy synthesis routes to achieve monophasic nanoparticles with high up conversion luminescence yield. Gadolinium phosphate $\left(\mathrm{GdPO}_{4}\right)$ is a very important host material for phosphor for Stokes shifted luminescence and has high thermal and chemical stability because $\mathrm{Gd}^{3+}$ has a half-filled $4 \mathrm{f}$ electron shell with a stable structure. ${ }^{5}$ Lanthanide phosphates are considered excellent host for rare earth (RE) doping and $\mathrm{RE}$ ion-doped $\mathrm{LnPO}_{4}$ phosphors and their nanoparticles have been investigated intensively. ${ }^{6-8}$ Recent work on

CSIR-National Physical Laboratory, Dr. K.S. Krishnan Road, New Delhi-110012, India. E-mail: santa@nplindia.org; Fax: +91 11 45609310; Tel: +91 1145609242

$\uparrow$ Electronic supplementary information (ESI) available. See DOI: 10.1039/c4ra04795h
RE doped $\mathrm{GdPO}_{4}$ nanophosphors has shown UC luminescence in $\mathrm{Tb}^{3+}$ and $\mathrm{Yb}^{3+}$ co-doped hexagonal and monoclinic $\mathrm{GdPO}_{4}$ nanoparticles ${ }^{9}$ and dual-modal bio-optical and magnetic resonance imaging properties. ${ }^{\mathbf{1 0}}$

$\mathrm{GdPO}_{4}$ is of particular interest for doping rare earth elements as it can act as efficient energy converter from IR to visible as well as UV to visible i.e., up conversion and downconversion. ${ }^{\mathbf{1 1 - 1 7}}$ In the present work, for UC emission $\mathrm{Ho}^{3+}$ is chosen as it can emit in green/red with sensitizer ion $\mathrm{Yb}^{3+}$ to augment the UC process since absorption cross section of $\mathrm{Yb}^{3+}$ for IR radiation is much higher than $\mathrm{Ho}^{3+}$. We describe the experimental investigation of photon upconversion in $\mathrm{GdPO}_{4}$ doped with $\mathrm{Ho}^{3+}$ and $\mathrm{Yb}^{3+}$ ions for the monoclinic phase nanoparticles synthesized through various routes. We have shown that we can achieve double RE ion doped monophasic nanocrystals through various synthesis routes that exhibit synthesis route dependent emission colour under IR excitation. The intense red/green emission from the nanophosphor shows great promise and can be used in IR-sensors, LEDs and as solar spectrum converters.

\section{Experiment}

We prepared $\mathrm{Gd}_{(84 \%)} \mathrm{PO}_{4}: \mathrm{Ho}_{(1 \%)}, \mathrm{Yb}_{(15 \%)}$ phosphors by four different methods, including SSR, CPP, SG and ACP.

Analytical grade reagents were used as reactants without further purification. Gadolinium(III) oxide $\left(\mathrm{Gd}_{2} \mathrm{O}_{3}\right.$, Specpure, 99.99\%), holmium(III) oxide $\left(\mathrm{Ho}_{2} \mathrm{O}_{3}\right.$, Specpure, 99.99\%), and ammonium dihydrogen phosphate $\left(\left(\mathrm{NH}_{4}\right)_{2} \mathrm{HPO}_{4}\right.$, Specpure, 99.999\%) and ytterbium oxide $\left(\mathrm{Yb}_{2} \mathrm{O}_{3}\right.$, Specpure, 99.99\%), were used as precursors. Nitric acid (Merck, p.a.) and ammonia 
solution (Merck, p.a.), ethanol (A.R.) were used throughout the experiment.

The stoichiometric reactants and boric acid ( 3 wt $\%)$ were mixed and ground thoroughly in an agate mortar, packed in recrystallized alumina boat and calcined at $1200{ }^{\circ} \mathrm{C}$ for $2 \mathrm{~h}$ in an air atmosphere. After calcining, the products were reground into white powders and the $\mathrm{Ho}^{3+}, \mathrm{Yb}^{3+}$ activated $\mathrm{GdPO}_{4}$ phosphor was obtained.

For co-precipitation synthesis the stoichiometric quantities of all the oxide precursors were dissolved in minimal amount of diluted nitric acid $\left(\mathrm{HNO}_{3}\right)$ to a transparent solution of nitrate salts, adjusting to $\mathrm{pH} 8$ with ammonia solution $\left(\mathrm{NH}_{4} \mathrm{OH}\right)$ and hydrogen peroxide $\left(\mathrm{H}_{2} \mathrm{O}_{2}\right)$ in the volume ratio $3: 1$, when ultrafine particles of $\mathrm{GdPO}_{4}$ doped with $\mathrm{RE}$ started forming. ${ }^{18}$ The resulting nanocrystals of $\mathrm{GdPO}_{4}: \mathrm{Ho}^{3+}, \mathrm{Yb}^{3+}$ were separated from the solution by filtering and then washed repeatedly with DI water and ethanol to remove soluble as well as surface bound impurities followed by drying at $60{ }^{\circ} \mathrm{C}$.

The third method that we adopted for synthesis of $\mathrm{Gd}_{0.84} \mathrm{PO}_{4}: \mathrm{Ho}_{0.01} \mathrm{Yb}_{0.15}$ nanoparticles is pechini-type SG method, adopted from Z. Xia et.al. ${ }^{19}$ The stoichiometric amount of precursors i.e. $\mathrm{Gd}_{2} \mathrm{O}_{3}, \mathrm{Eu}_{2} \mathrm{O}_{3}$ and $\left(\mathrm{NH}_{4}\right)_{2} \mathrm{HPO}_{4}$ were dissolved in diluted $\mathrm{HNO}_{3}$ (A.R.) under vigorous stirring at $80{ }^{\circ} \mathrm{C}$ for 15 minutes to make metal nitrate salts, then a water-ethanol $\left(V_{\mathrm{w}} / V_{\mathrm{e}}=1: 7\right)$ solution was added. Citric acid ( $\left.3 \mathrm{~mol}\right)$ was added as chelating agent of metal ions and polyhydroxy alcohol (ethylene glycol, $6 \mathrm{~mol}$ ) as a cross-linking agent. The transparent solution after stirring for 15-20 minutes turned to slightly brown sol which was further heated at $80^{\circ} \mathrm{C}$ for an hour. The final product was obtained as white powder which was then washed and dried at $150{ }^{\circ} \mathrm{C}$ for $12 \mathrm{~h}$. The sol gel synthesis was employed as it can ensure uniformity of composition and lessen segregation of particular metal ions.

In the fourth approach of synthesis by auto-combustion process, we dissolved the stoichiometric quantities of precursors in nitric acid diluted with deionised water. Nitrate acts as an oxidizer for the fuel during the combustion reaction. Urea $\left(\mathrm{NH}_{2} \mathrm{CONH}_{2}\right)$ is an attractive fuel for originating the formation of powders with crystallite sizes in the submicron/ nanosized range and act as a complexing agent for metal ions because it contains two amino groups located at the extremes of its chemical structure. The oxidants and the fuels were mixed in the required molar ratios in a minimum volume of de-ionized water to obtain transparent aqueous solutions. These solutions after thermal dehydration (at $\sim 80^{\circ} \mathrm{C}$ on a hot plate to remove the excess solvent) gave highly viscous liquids. As soon as the viscous liquids were formed, the temperature of the hot plate was increased to $250^{\circ} \mathrm{C}$. At this stage, the viscous liquid swelled and auto-ignited, with the rapid evolution of large volume of gases, to produce voluminous powders which upon cooling was ground to obtain pure and well crystalline powders.

A part of samples prepared by CPP, ACP and SG method were annealed in air, at temperature $1000{ }^{\circ} \mathrm{C}$, for 2 hours to investigate the effect on crystallinity and luminescence efficiency.

\section{Results and discussion}

The X-ray Diffraction (XRD) pattern of the annealed $\mathrm{GdPO}_{4}: \mathrm{Ho}$, $\mathrm{Yb}$ powder samples are presented in Fig. 1 including a reference diffractogram of a $\mathrm{GdPO}_{4}$ bulk powder. All characteristic peaks for $\mathrm{GdPO}_{4}: \mathrm{Ho}$, Yb marked by their indices i.e. ( $\left.\begin{array}{lll}\overline{1} & 1 & 1\end{array}\right),\left(\begin{array}{lll}0 & 2 & 0\end{array}\right)$, ( $\left.\begin{array}{lll}2 & 0 & 0\end{array}\right),\left(\begin{array}{lll}1 & 2 & 0\end{array}\right),\left(\begin{array}{lll}2 & 0 & 2\end{array}\right),\left(\begin{array}{lll}1 & 1 & 2\end{array}\right),\left(\begin{array}{lll}3 & 1 & 1\end{array}\right)$, matched well with the standard JCPDS data (32-0386) of monoclinic phase. No obvious XRD peaks arising from impurities were found; meaning that pure phase for all the samples can be successfully achieved through different synthesis routes followed by annealing. XRD pattern of as synthesized $\mathrm{GdPO}_{4}$ samples are shown in the inset of Fig. 1. $\mathrm{GdPO}_{4}: \mathrm{Ho}, \mathrm{Yb}$ as synthesized by ACP method displays one broad peak centred at $2 \theta \approx 29.68^{\circ}$, which is identified as the $\left(\begin{array}{lll}1 & 2 & 0\end{array}\right)$ reflection of monoclinic phase of $\mathrm{GdPO}_{4}$. For as synthesized $\mathrm{GdPO}_{4}$ samples prepared via CPP and SG method, sharp peaks corresponding to both hexagonal (JCPDS card 390232) and monoclinic (JCPDS card 32-0386) phases were obtained indicating formation of mixed phase. As hexagonal to monoclinic phase transformation of $\mathrm{GdPO}_{4}$ occurs due to annealing at $1000{ }^{\circ} \mathrm{C}$ for 2 hours, monophasic monoclinic nanocrystals were obtained after annealing.

Transmission electron microscopy (TEM) \& HRTEM were used to characterize the synthesized material concerning the crystal structure, shape, size and size distribution of the nanoparticles. The TEM images show particle distribution with different morphology (see ESI $\dagger$ ). High resolution TEM image of $\mathrm{GdPO}_{4}: \mathrm{Ho}$, Yb sample made by SSR method (Fig. 2(i)) shows well defined aggregated crystalline particles below $50 \mathrm{~nm}$ with irregular shapes. The selected area electron diffraction (SAED) pattern presented in Fig. 2(ii) confirms its crystallinity. In the SAED pattern of SSR sample (Fig 2(ii)) the diffraction spots correspond to (l $\left.\begin{array}{lll}0 & 2 & 0\end{array}\right),\left(\begin{array}{lll}2 & 0 & 0\end{array}\right)$ and ( $\left(\begin{array}{lll}0 & 1 & 2\end{array}\right)$ planes of monoclinic phase of $\mathrm{GdPO}_{4}$ (JCPDS-32-0386) as also seen in XRD pattern

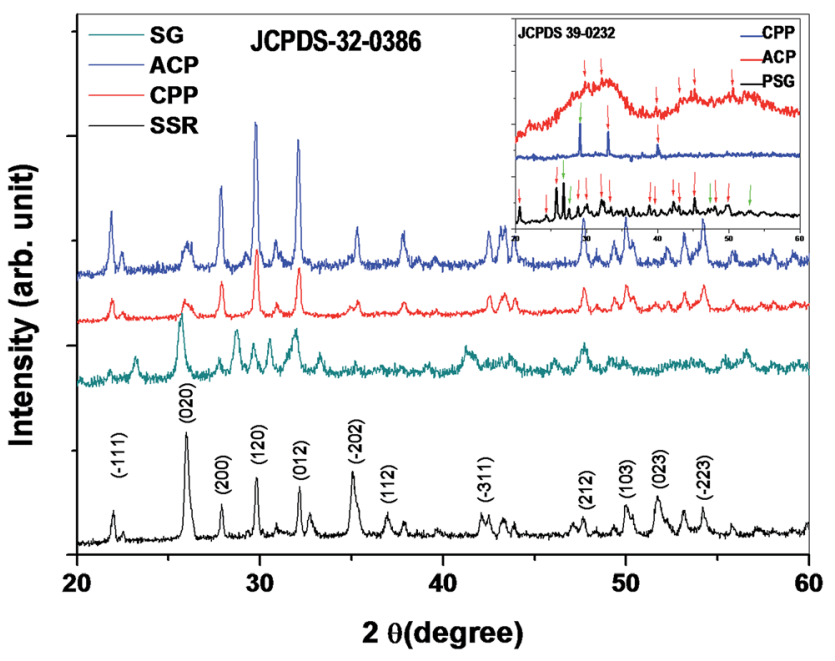

Fig. 1 XRD patterns of annealed $\left(1000{ }^{\circ} \mathrm{C}\right) \mathrm{GdPO}_{4}: \mathrm{Ho}^{3+}, \mathrm{Yb}^{3+}$ samples by auto combustion process, pechini type sol-gel, CPP and SSR method, inset shows the XRD spectra of as prepared samples by ACP, CPP and SG method, green and red arrow respectively indicate diffraction peaks corresponding to monoclinic and hexagonal phase. 


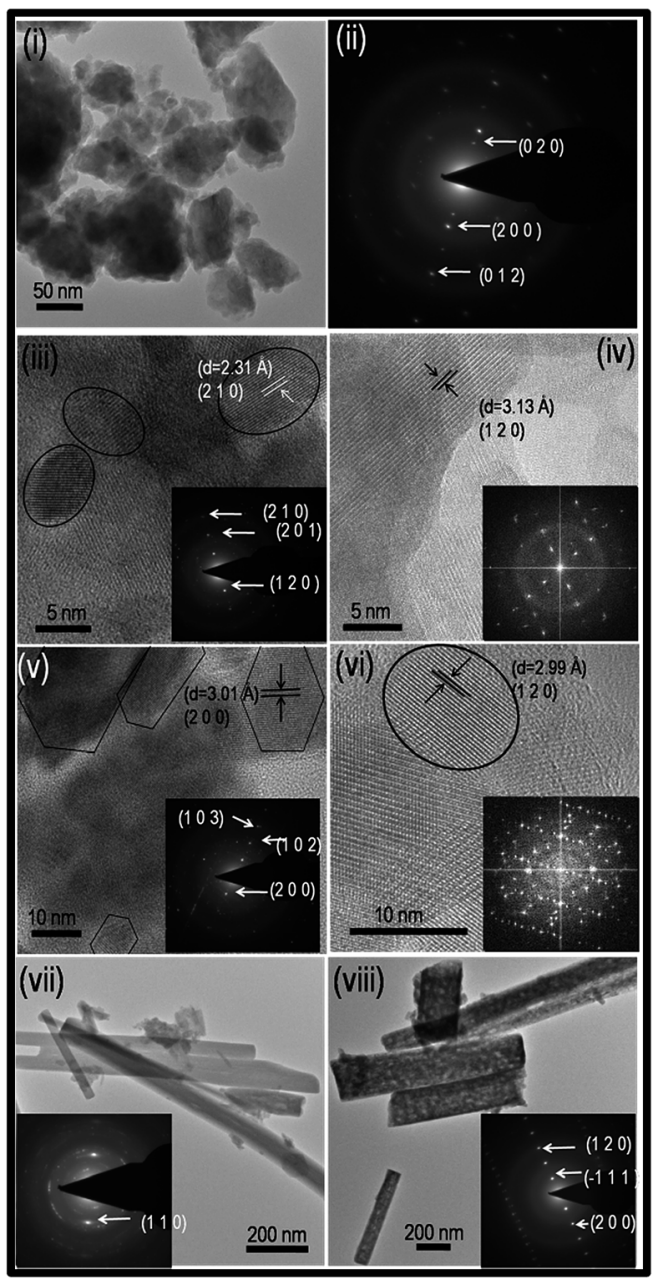

Fig. 2 (i \& ii) TEM image and SAED pattern of SSR sample respectively; (iii) HRTEM image of as prepared CPP sample and in inset its SAED pattern; (iv) TEM images of annealed CPP sample with its FFT image in inset; (v) HRTEM image of as prepared ACP sample and in inset its SAED pattern; (vi) HRTEM images of annealed ACP sample with its FFT image in inset; (vii) TEM image of as prepared SG sample and in inset its SAED pattern; (viii) TEM images of annealed SG sample with its SAED pattern image in inset.

(Fig. 1). The HRTEM image of CPP derived sample Fig. 2(iii) shows the projection of more or less elliptical shaped crystallites with an estimated size of 5-10 $\mathrm{nm}$. Lattice fringes shown in HRTEM image of as prepared CPP sample (Fig. 2(iii)) corresponds to interplanar spacing $(d=2.31 \AA)$ and $h k l$ plane $\left(\begin{array}{lll}2 & 1 & 0\end{array}\right)$, which matches hexagonal phase conforming to the XRD pattern (inset Fig. 1). The elliptical shape of nanoparticles changed to elongated particle with very good crystallinity as revealed by HRTEM image that shows well formed lattice fringes with interplanar spacing $(d=3.13 \AA)$ corresponding to $\left(\begin{array}{lll}1 & 2 & 0\end{array}\right)$ planes of monoclinic phase, conforming to XRD pattern (Fig. 1). Fig. 2(v) displays the HRTEM image of $\mathrm{GdPO}_{4}: \mathrm{Ho}, \mathrm{Yb}$ nanoparticles prepared via auto-combustion process. It can be seen from Fig. 2(v) that the nanoparticles show hexagonal shape (with particle sizes of about $20-30 \mathrm{~nm}$ ) in consistence with XRD results. The lattice fringes of nanoparticles of as prepared ACP sample (Fig. 2(v)), indicate interplanar spacing of $d=3.01 \AA$ which corresponds to $\left(\begin{array}{lll}2 & 0 & 0\end{array}\right)$ planes of hexagonal lattice of $\mathrm{GdPO}_{4}$ (JCPDS card 39-0232). Though XRD pattern (inset Fig. 1) indicate broad peek corresponding to hexagonal phase, the SAED pattern show diffraction spots corresponding to $h \mathrm{kl}$ planes ( $\left(\begin{array}{lll}2 & 0 & 0\end{array}\right),\left(\begin{array}{lll}1 & 0 & 3\end{array}\right)$ and $\left(\begin{array}{lll}1 & 0 & 2\end{array}\right)$ which confirm formation of hexagonal phase in as prepared sample. The sample undergo phase transformation to monoclinic phase after annealing as evidenced by HRTEM pattern (Fig. 2(vi)), well formed lattice fringes corresponding to $h k l$ planes $\left(\begin{array}{lll}2 & 2\end{array}\right)$ with interplanar spacing 2.99 A. Corresponding FFT pattern shown in inset Fig. 2(vi) exhibit well defined spots. The TEM image of SG sample shown in Fig. 2(vii), exhibit well formed rod shaped particles with width $\sim 50-100 \mathrm{~nm}$ and few micrometer in length. After calcination at $1000{ }^{\circ} \mathrm{C}$ for $2 \mathrm{~h}$, the rods broaden and become thick due to the growth of particles in different directions. Formation of well crystalline nanorods by SG is a very good achievement since this method produces large yield. And morphological homogeneity was better for CPP and SG derived samples than the SSR and ACP derived samples. Fig 2(vii) inset show the SAED pattern of as prepared SG sample, formation of ring pattern with well defined diffraction spots indicate its highly crystalline nature and diffraction spot corresponds to $h \mathrm{kl}$ plane $\left(\begin{array}{lll}1 & 1 & 0\end{array}\right)$ with interplanar spacing $3.45 \AA$ correspond to the observed prominent hexagonal peak in XRD pattern (Fig. 1 inset). Diffraction spots in annealed

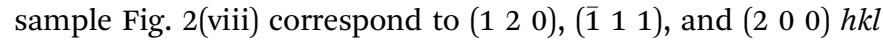
planes of monoclinic phase, which are also experienced as prominent peak in XRD pattern (Fig. 1).

Thus the morphology of the nanoparticles was different for the all four preparation methods-SSR, CPP, ACP and SG. It is also observed that large amount of rare earth dopant ions in substitutional positions of $\mathrm{Gd}^{3+}$ do not interfere with the crystalline phase formation indicated by their XRD and HRTEM results which do not show any precipitated phase of the rare earth ions.

The UC emission spectra of the $\mathrm{GdPO}_{4}$ doped with $\mathrm{Ho}^{3+}, \mathrm{Yb}^{3+}$ measured at room temperature under NIR excitation $(980 \mathrm{~nm})$ is shown in Fig. 3. In nanocrystals prepared by SSR method, the emission spectrum exhibited a weak band in the green due to

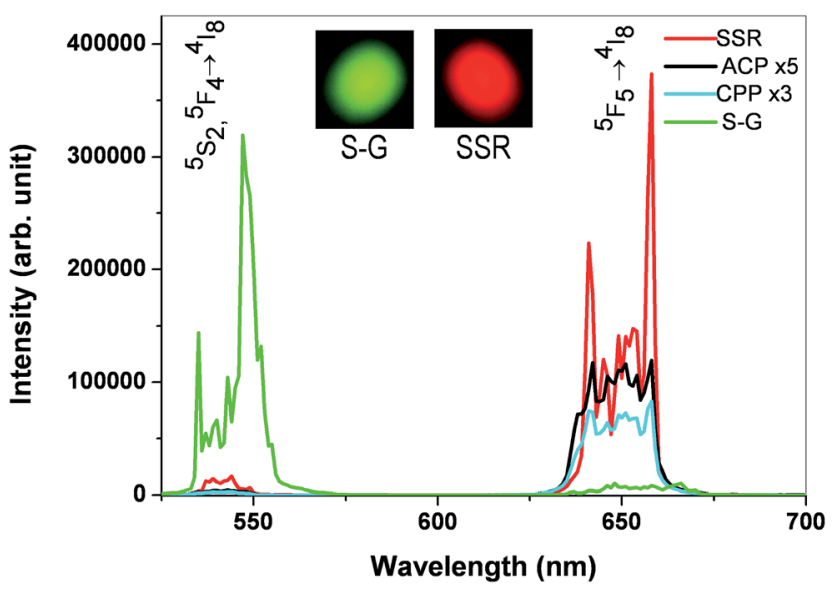

Fig. 3 UC PL emission spectra of $\mathrm{GdPO}_{4}: \mathrm{Ho}^{3+}, \mathrm{Yb}^{3+}$ samples prepared by all four synthesis protocol, insets show actual photographs of powder sample under $980 \mathrm{~nm}$ diode laser. 


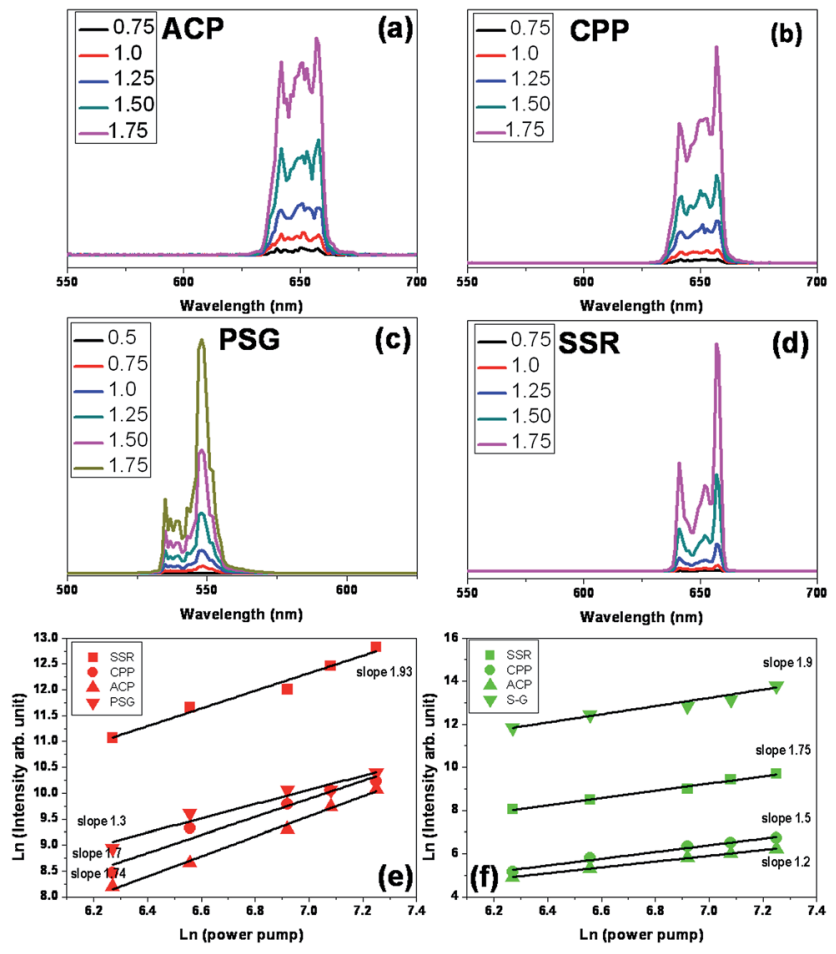

Fig. 4 (a-d) UC PL emission spectra at different pump power, numbers in the inset are the pump laser power in Watts and (e and f) dependence of green and red UC emission intensity on pump power of the NIR laser $(980 \mathrm{~nm})$ of $\mathrm{GdPO}_{4}: \mathrm{Ho}^{3+}, \mathrm{Yb}^{3+}$ samples prepared by four different synthesis processes.

${ }^{5} \mathrm{~S}_{2},{ }^{5} \mathrm{~F}_{4} \rightarrow{ }^{5} \mathrm{I}_{8}$ transition at $547 \mathrm{~nm}$ and strong sharp peaks in the red at about 640 and $657 \mathrm{~nm}$ due to the ${ }^{5} \mathrm{~F}_{5} \rightarrow{ }^{5} \mathrm{I}_{8}$ transition of $\mathrm{Ho}^{3+}$. The prominent $657 \mathrm{~nm}$ emission of $\mathrm{Ho}^{3+}$ ion in $\mathrm{GdPO}_{4}$ material is very promising for application as a red UC phosphor for IR sensing devices. Whereas the emission spectra for annealed samples prepared by CPP and ACP method were exactly the same as emission spectra of SSR sample but with much less intensity. In contrast, nanorods prepared by SG process, exhibit intense green band around $547 \mathrm{~nm}$ arising from $\left({ }^{5} \mathrm{~F}_{4},{ }^{5} \mathrm{~S}_{2}\right) \rightarrow{ }^{5} \mathrm{I}_{8}$ transition and much less red emission at $657 \mathrm{~nm}$. The photograph of the samples under IR laser $(980 \mathrm{~nm})$ excitation is shown as inset in Fig. 3.

In order to understand the multiphoton absorption process in UC emission, dependence of pump laser power on UC emission intensity was measured for all four samples. The variation of UC PL emission intensity of the four samples prepared through different synthesis routes with exciting IR laser $(980 \mathrm{~nm})$ power are shown in Fig. 4(a-d) and clearly reveals the synthesis route dependent spectral variation of the UC emission. Fig. 4(e and f) show the dependence of integrated upconverted emissions on IR excitation power. The slopes of the lines are $\approx 2$, as expected for two-photon excitations. However, it has been shown recently that the intensity of up-converted luminescence, excited by sequential absorption of $n$ photons, has a dependence on absorbed power $P$, which may range between limits of $P^{n}$ and $P^{1} \cdot{ }^{16}$ Laser pump power $v s$. UC emission intensity plots indicate the values of slopes for the green emission at $547 \mathrm{~nm}$, and the red emission at $657 \mathrm{~nm}$ in Fig. 4(a and $\mathrm{b})$. The predominant IR excitation mechanism is through ground state excitation of two $\mathrm{Yb}^{3+}$ ions to ${ }^{2} \mathrm{~F}_{5 / 2}$ level by absorption of two IR photons and energy transfer to $\mathrm{Ho}^{3+}$ ions exciting it from ${ }^{5} \mathrm{I}_{8}$ ground state to intermediate excited state ${ }^{5} \mathrm{I}_{6}$. This process is followed by interaction of another excited $\mathrm{Yb}^{3+}$ ion which elevates the excited $\mathrm{Ho}^{3+}$ ion from ${ }^{5} \mathrm{I}_{6}$ state into the emitting ${ }^{5} \mathrm{~S}_{2}$ level as $980 \mathrm{~nm}$ photon energy matches with the level spacing of ${ }^{5} \mathrm{I}_{6}-{ }^{5} \mathrm{~S}_{2}$ transition. ${ }^{20} \mathrm{Ho}^{3+}$ ions in the ${ }^{5} \mathrm{~S}_{2},{ }^{5} \mathrm{~F}_{4}$ levels can relax to ground state ${ }^{5} \mathrm{I}_{8}$ by emission of green photons. The red UC emission from $\mathrm{Ho}^{3+}$ occurs due to transition from excited ${ }^{5} \mathrm{~F}_{5}$ level to ground ${ }^{5} \mathrm{I}_{8}$ level. The red emitting ${ }^{5} \mathrm{~F}_{5}$ level can be populated either due to direct excited state absorption of one $980 \mathrm{~nm}$ photon from ${ }^{5} \mathrm{I}_{7}$ which is used as a bridge level that is populated by multiphonon relaxation from ${ }^{5} \mathrm{I}_{6}$ or through phonon relaxation from ${ }^{5} \mathrm{~S}_{2},{ }^{5} \mathrm{~F}_{4}$ levels. This

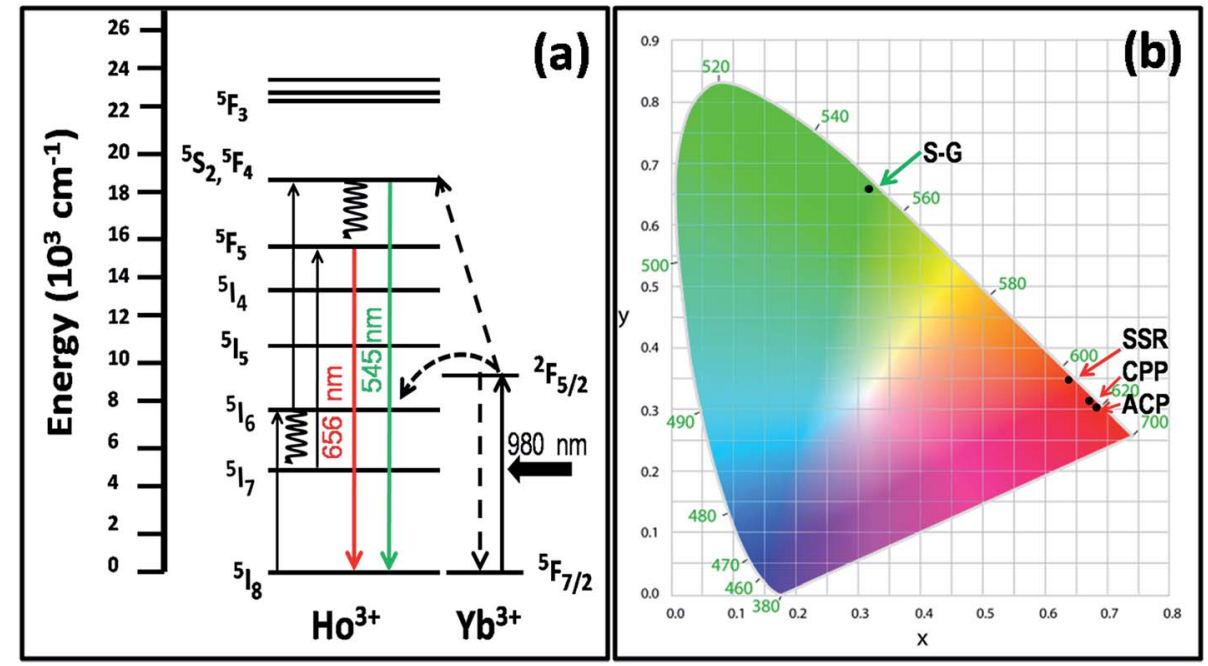

Fig. 5 (a) Energy level diagram showing excitation and charge transfer processes leading to UC luminescence emission from $\mathrm{Gd}^{3+}$ and $\mathrm{Ho}^{3+}$ ions in $\mathrm{GdPO}_{4}$; (b) $\mathrm{ClE}$ chromaticity diagram of different $\mathrm{GdPO}_{4}: \mathrm{Ho}^{3+}, \mathrm{Yb}^{3+}$ nanophosphor. 
indicates that two photon absorption is involved in RE ion excitation process from ground state to various excited levels of $\mathrm{Ho}^{3+}$ ions and different multiphonon relaxation paths lead to different population of the emitting level giving rise to UC emission predominantly in either green or red. The excitation and emission processes are explained in the energy level scheme depicted in Fig. 5(a).

The emission colour of UC luminescence of all the $\mathrm{GdPO}_{4}$ :$\mathrm{Ho}^{3+}, \mathrm{Yb}^{3+}$ nanoparticles have been calculated from the respective emission spectra and represented as the CIE coordinates (Fig. 5(b)). The colour coordinates of SSR, CPP, \& ACP synthesized samples fall in the red region and chromaticity coordinates are $(0.65,0.35),(0.67,0.33)$ and $(0.68,0.32)$ respectively. The $\mathrm{GdPO}_{4}: \mathrm{Ho}^{3+}, \mathrm{Yb}^{3+}$ samples prepared through SG method emits green light and its chromaticity coordinates are $(0.34,0.66)$. The chromaticity diagram (Fig. 5(b)) clearly indicate that due to change in synthesis route for the same lanthanide doping in the same host, the emission colour changes under IR excitation and varies from intense red to green.

\section{Conclusions}

We have synthesised intense green emitting UC phosphor $\left(\mathrm{GdPO}_{4}: \mathrm{Ho}^{3+}, \mathrm{Yb}^{3+}\right)$ by SG method and red emission via other route i.e. CPP, SSR and ACP under $980 \mathrm{~nm}$ IR excitation. The average size of the particles is less than $50 \mathrm{~nm}$ in diameter and varied from nanoparticles to well formed nanorods depending upon the synthesis process adopted. We have measured the upconversion emission spectra of each sample, synthesized by four different routes, under a $980 \mathrm{~nm}$ laser at different excitation power and plotted the pump power $v s$. UC emission intensity to obtain the relationship of number of photons involved in the UC process for each sample for both green and red emission. The energy level diagram is presented as per the multi photon process inferred for experimental observation of the $\mathrm{Ho}, \mathrm{Yb}$ system. The calculated colour coordinates for UC emission of each sample plotted in the CIE chromaticity diagram clearly shows the synthesis route dependent tunability of emission. Such upconverting nanocrystals have promising applications in security ink, IR sensors and solar spectrum conversion.

\section{Acknowledgements}

This present work was supported by the TAPSUN project under CSIR Solar Mission program of India. We thank Dr Sukhvir Singh for TEM and Dr VPS Awana for XRD measurements.

\section{References}

1 H. Wang, C. K. Duan and P. A. Tanner, J. Phys. Chem. C, 2008, 112, 16651-16654.

2 H. Guo, F. Li, R. F. Wei, H. Zhang and C. G. Ma, J. Am. Ceram. Soc., 2012, 95(4), 1178-1181.

3 K. Kompe, H. Borchert, J. Storz, A. Lobo, S. Adam, T. Moller and M. Haase, Angew. Chem., Int. Ed., 2003, 42, 5513-5516.

4 M. Yu, H. Wang, C. K. Lin, G. Z. Li and J. Lin, Nanotechnology, 2006, 17, 3245-3252.

5 C. Cao, H. K. Yang, B. K. Moon, B. C. Choi and J. H. Jeong, J. Electrochem. Soc., 2011, 158, 2.

6 J. Zhang, C. Mi, H. Wu, H. Huang, C. Mao and S. Xua, Anal. Biochem., 2012, 15, 673-679.

7 J. Ryu, H. Y. Park, K. Kim, H. Kim, J. H. Yoo and M. Kang, J. Phys. Chem. C, 2010, 114, 21077-21082.

8 Q. Cheng, J. Sui and W. Cai, Nanoscale, 2012, 4, 779-784.

9 Z. Xuedong, H. Tao, B. Xiaoyi, et al., Integr. Ferroelectr., 2013, 146, 115-121.

10 R. Wenlu, T. Gan, Z. Liangjun, et al., Nanoscale, 2012, 4, 3754-3760.

11 T. Grzyb, A. Gruszeczka, R. J. Wiglusz, Z. Sniadecki, B. Idzikowski and S. Lis, J. Mater. Chem., 2012, 22, 2298922997.

12 S. Lu, J. Zhang, J. Zhang, H. Zhao, Y. Luo and X. Ren, Nanotechnology, 2010, 21, 365709.

13 L. Yu, D. Li, M. Yue, J. Yao and S. Lu, Chem. Phys., 2006, 326, 478-482.

14 N. K. Sahu, R. S. Ningthoujam and D. Bahadur, J. Appl. Phys., 2012, 112, 014306.

15 V. Kumar, S. Singh, R. K. Kotnala and S. Chawla, J. Lumin., 2014, 146, 486-491.

16 M. Evangelisti, T. G. Sorop, O. N. Bakharev, D. Visser, A. D. Hillier, J. J. Alonso, M. Haase, L. A. Boatner and L. J. Jongh, Phys. Rev. B: Condens. Matter Mater. Phys, 2011, 84, 094408.

17 W. Ryba-Romanowski, S. Golab, G. Dominiak-Dzik, P. Solarz and T. Lukasiewicz, Appl. Phys. Lett., 2001, 79, 5.

18 V. Kumar, A. F. Khan and S. Chawla, J. Phys. D: Appl. Phys., 2013, 46, 365101.

19 Z. Xia, D. Chen, M. Yang and T. Ying, J. Phys. Chem. Solids, 2010, 71, 175-180.

20 S. Wang, S. Song, R. Deng, H. Guo, Y. Lei, F. Cao, X. Li, S. Su and H. Zhang, CrystEngComm, 2010, 12, 3537. 